OPEN ACCESS

Edited by:

Benoit Chassaing, Georgia State University,

United States

Reviewed by: Jennifer Auchtung, University of Nebraska-Lincoln,

United States

Alison Vrbanac,

Siolta Therapeutics,

United States

Nathalie Rolhion,

Institut National de la Santé et de la

Recherche Médicale (INSERM),

France

*Correspondence:

Prithvi Raj

prithvi.raj@utsouthwestern.edu

Specialty section:

This article was submitted to Microbiome in Health and Disease,

a section of the journal

Frontiers in Cellular and

Infection Microbiology

Received: 09 March 2021

Accepted: 14 June 2021

Published: 01 October 2021

Citation:

Zhang B, Brock M, Arana C, Dende C, van Oers $N$, Hooper $L V$ and Raj $P$ (2021) Impact of Bead-Beating Intensity on the Genus- and Species-Level

Characterization of the Gut Microbiome Using Amplicon and Complete

16S rRNA Gene Sequencing.

Front. Cell. Infect. Microbiol. 11:678522.

doi: 10.3389/fcimb.2021.678522

\section{Impact of Bead-Beating Intensity on the Genus- and Species-Level Characterization of the Gut Microbiome Using Amplicon and Complete 16S rRNA Gene Sequencing}

\author{
Bo Zhang ${ }^{1,2}$, Matthew Brock ${ }^{1,2}$, Carlos Arana ${ }^{1,2}$, Chaitanya Dende ${ }^{1}$, \\ Nicolai Stanislas van Oers ${ }^{1}$, Lora V. Hooper ${ }^{1}$ and Prithvi Raj ${ }^{1,2 *}$

\begin{abstract}
1 Department of Immunology, University of Texas Southwestern Medical Center, Dallas, TX, United States, ${ }^{2}$ Microbiome TX, United States
\end{abstract} \\ Research Laboratory (MRL), Department of Immunology, University of Texas Southwestern Medical Center, Dallas,
}

Bead-beating within a DNA extraction protocol is critical for complete microbial cell lysis and accurate assessment of the abundance and composition of the microbiome. While the impact of bead-beating on the recovery of OTUs at the phylum and class level have been studied, its influence on species-level microbiome recovery is not clear. Recent advances in sequencing technology has allowed species-level resolution of the microbiome using full length 16S rRNA gene sequencing instead of smaller amplicons that only capture a few hypervariable regions of the gene. We sequenced the v3-v4 hypervariable region as well as the full length 16S rRNA gene in mouse and human stool samples and discovered major clusters of gut bacteria that exhibit different levels of sensitivity to bead-beating treatment. Full length 16S rRNA gene sequencing unraveled vast species diversity in the mouse and human gut microbiome and enabled characterization of several unclassified OTUs in amplicon data. Many species of major gut commensals such as Bacteroides, Lactobacillus, Blautia, Clostridium, Escherichia, Roseburia, Helicobacter, and Ruminococcus were identified. Interestingly, v3-v4 amplicon data classified about $50 \%$ of Ruminococcus reads as Ruminococcus gnavus species which showed maximum abundance in a 9 min beaten sample. However, the remaining $50 \%$ of reads could not be assigned to any species. Full length $16 \mathrm{~S}$ rRNA gene sequencing data showed that the majority of the unclassified reads were Ruminococcus albus species which unlike $R$. gnavus showed maximum recovery in the unbeaten sample instead. Furthermore, we found that the Blautia hominis and Streptococcus parasanguinis species were differently sensitive to bead-beating 
treatment than the rest of the species in these genera. Thus, the present study demonstrates species level variations in sensitivity to bead-beating treatment that could only be resolved with full length $16 \mathrm{~S}$ rRNA sequencing. This study identifies species of common gut commensals and potential pathogens that require minimum (0-1 min) or extensive (4-9 min) bead-beating for their maximal recovery.

Keywords: microbiome, 16S, sequencing, DNA extraction, bead-beating, short-read, long-read

\section{INTRODUCTION}

Trillions of symbiotic microbial cells are present in and on the human body that constitute human microbiota (Huttenhower et al., 2021). The microbiome refers to the collection of genes in these microorganisms. Specific clusters of these microbes in various body parts constitute the organ-specific microbiome, for example microbial communities in the gut constitute the gut microbiome. The microbiome includes a variety of organisms, i.e., bacteria, yeasts, fungi, protozoa, and viruses (Matijašić et al., 2020). However, bacteria comprise the vast majority of these microorganisms which play a critical role in the breakdown and absorption of nutrients, sugars, and proteins that humans cannot otherwise digest and metabolize on their own to synthesize essential amino acids and vitamins. Microbiome profiling assays typically sequence polymorphisms in the 16S rRNA gene of bacteria, the 18S rRNA gene of eukaryotes, and ITS regions in the case of fungi to infer taxonomic classification of the microbiome (Janda and Abbott, 2007; Nash et al., 2017). Literature suggests that DNA extraction methods significantly impact the microbiome study results (Costea et al., 2017; Sinha et al., 2017). Many studies have optimized protocols to extract microbial DNA from different types of samples to use as a template for 16S rRNA gene sequencing (Gill et al., 2006; Nelson et al., 2010; Werner et al., 2012; Falony et al., 2016; The Integrative HMP (iHMP) Research Network Consortium, 2019). A number of prior studies provide evidence that methods of sample collection, storage, and DNA extraction are critical for accurate profiling of microbiota in environmental (Baker et al., 2003; Tremblay et al., 2015; Bag et al., 2016) or human samples (Wu et al., 2010; Momozawa et al., 2011; Willner et al., 2012; Brooks et al., 2015; Costea et al., 2017; Sinha et al., 2017). Literature suggests that complete lysis of the bacterial cell wall is critical for optimum yield of high integrity DNA for both short and long-read sequencing workflows (Jenkins et al., 2019). Lysis protocols include procedures that lead to physical and or enzymatic disruption of the microbial cell wall (Bag et al., 2016; Gill et al., 2016; Valentini et al., 2016). It has been observed that extended lysis time and mechanical disruption can enhance nucleic acid yield. However, extended mechanical lysis time can also reduce molecular complexity by excessive shearing of microbial DNA into smaller fragments (von Wintzingerode et al., 1997; Dilhari et al., 2017). Particularly, Gram-positive bacteria pose the greatest challenge for complete lysis due to their thick cell walls and complex composition (Kim et al., 2015).

Metagenomic analysis of the human microbiome shows that each individual can harbor hundreds of different bacterial species and varying lysis methods can impact their recovery (Qin et al., 2010; Gill et al., 2016). It is therefore very important to use a DNA extraction method that can optimally extract DNA from the entire bacterial community with minimal bias for downstream sequencing analysis. Current DNA extraction methods use various sample homogenization or lysis protocols, which can result in variability shown in microbiome profiles. Mechanical bead-beating or enzymatic cell lysis steps have been shown to be crucial for maximum DNA recovery from all kinds of organisms (de Boer et al., 2010). Bead-beating has become a common method of bacterial cell lysis in microbial metagenomics studies (Fiedorova et al., 2019). Here we assess the impact of bead-beating treatment on gut microbiome recovery using the v3-v4 amplicon and the full length $16 \mathrm{~S}$ rRNA sequencing method. We characterize genus- and species-level diversity in mouse and human stool and assess variation in OTU recovery pertaining to differential sensitivity to bead-beating treatment in the DNA extraction protocol.

\section{MATERIALS AND METHODS}

\section{Sample Collection}

We investigated five mouse (C57/Bl6) stool samples (designated as M1-M5), five human stool samples (designated as H1-H5), and one ZymoBIOMICS Gut Microbiome Standard (Cat\#D6331) from Zymo Research. The Zymo control sample is comprised of 21 different bacterial and fungal strains that mimic the human gut microbiome. Prior knowledge on the composition and proportions of various bacteria in this sample allowed for validation of our sequencing and data analysis pipeline. Stool samples were collected under sterile conditions and stored in DNA/RNA Shield, a nucleic acid stabilizing solution from Zymo Research (R1100). DNA/RNA Shield provides an accurate molecular signature of the sample at the time of collection by preserving nucleic acids at ambient temperature and inactivating organisms including infectious agents. Human stools were collected from healthy volunteers under UT Southwestern Institutional Review Board (IRB) Number STU-022011-211. All research protocols and experiment methods used in this study were approved by the IRB. All participants gave their written informed consent to participate in the research.

\section{Bead-Beating Condition and DNA Extraction Method}

We used the ZymoBIOMICS ${ }^{\mathrm{TM}}$ DNA Miniprep Kit (D4300) for DNA extraction on all the study samples. Figure 1 illustrates the design and experimental workflow of the study. To make sure 
that each aliquot received unbiased representation of the sample, the specimen was first hand-mixed using the spoon provided in the DNA/RNA Shield Fecal Collection Tubes. Then once all the large clumps were dissolved in the specimen and the sample appeared to be more uniform in solution, $1 \mathrm{ml}$ of aliquots were prepared for various bead-beating conditions. Similarly, $75 \mathrm{ul}$ of ZymoBIOMICS Gut Microbiome Standard (D6331) and $925 \mathrm{ml}$ of DNA/RNA Shield was aliquoted into each of four separate tubes to test with four bead-beating conditions. Each sample was aliquoted into a ZR BashingBead lysis tube $(0.1$ and $0.5 \mathrm{~mm}$ beads). Next, each sample tube was tightly closed and loaded onto the PowerLyzer 24 Homogenizer (110/220 V) for beadbeating. We selected four different bead-beating time points as illustrated in Figure 1: 0 min (no bead-beating at all), $1 \mathrm{~min}$ (one cycle of shaking for $1 \mathrm{~min}$ ), $4 \mathrm{~min}$ (two cycles of $2 \mathrm{~min}$ shaking, with a 2 min pause after each cycle), and 9 min ( 6 cycles of $1 \mathrm{~min}$ $30 \mathrm{~s}$, with a $2 \mathrm{~min}$ pause after each cycle). Each of these samples were bead-beaten at a speed of 2200 RPM and were maintained at a temperature of $20^{\circ} \mathrm{C}$ throughout the bead-beating process. Following beat-beating and lysis, DNA was purified using the
A

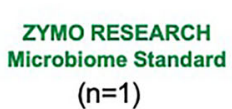
$(n=1)$
B Mouse stool
Sample
$(n=5)$
C

Human stool

Sample $(n=5)$

\begin{tabular}{|c|c|c|c|c|c|c|c|c|c|c|}
\hline ZYMO & \multicolumn{6}{|c|}{ MOUSE STOOL } & \multicolumn{4}{|c|}{ HUMAN STOOL } \\
\hline Z1 & M1 & M2 & M3 & M4 & M5 & H1 & H2 & H3 & H4 & H5 \\
\hline $0-$ Min & $0-\operatorname{Min}$ & 0-Min & 0-Min & $0-$ Min & 0-Min & 0-Min & 0-Min & $0-\operatorname{Min}$ & 0-Min & 0-Min \\
\hline 1-Min & 1-Min & 1-Min & 1-Min & 1-Min & 1-Min & 1-Min & 1-Min & 1-Min & 1-Min & 1-Min \\
\hline 4-Min & 4-Min & 4-Min & 4-Min & 4-Min & 4-Min & 4-Min & 4-Min & 4-Min & 4-Min & 4-Min \\
\hline 9-Min & 9-Min & 9-Min & 9-Min & 9-Min & 9-Min & 9-Min & 9-Min & 9-Min & 9-Min & 9-Min \\
\hline
\end{tabular}

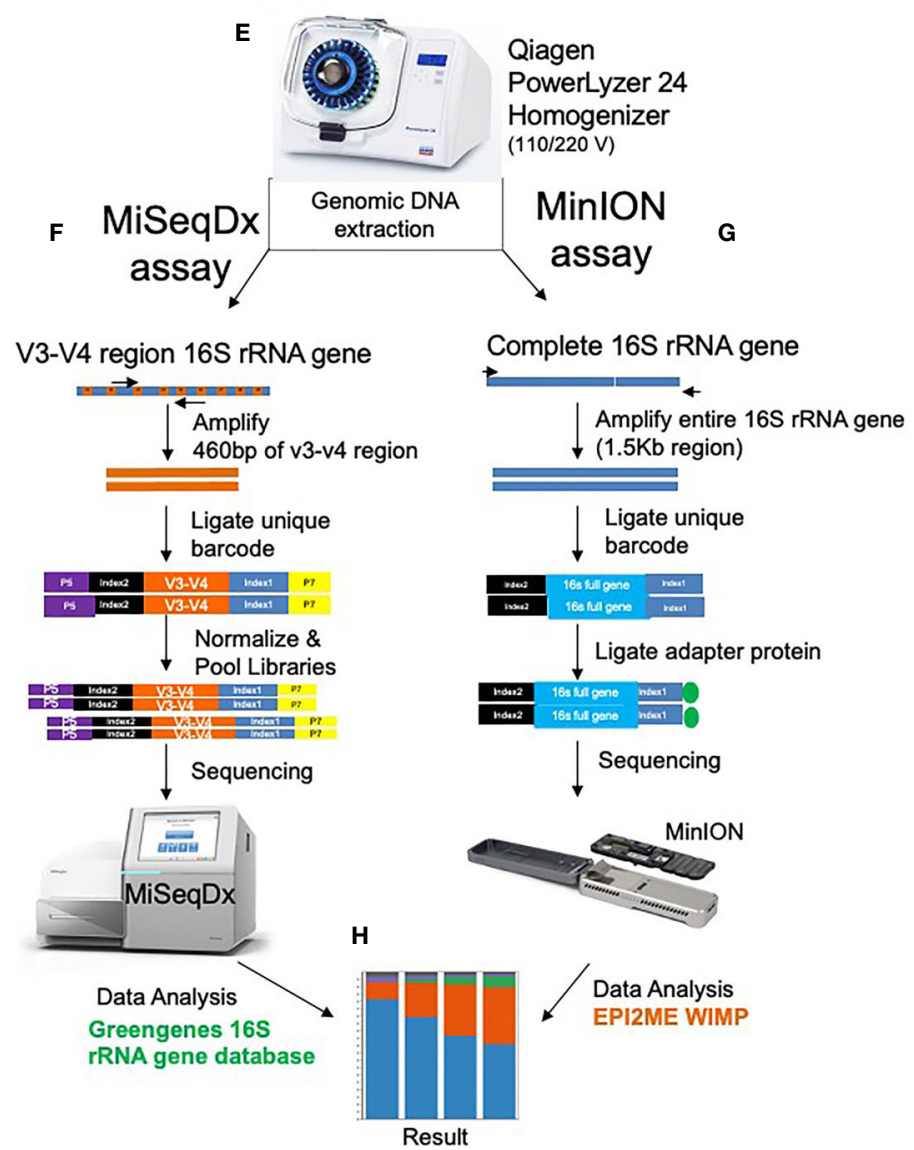

FIGURE 1 | Illustration of the experimental design and workflow. One Zymo human gut microbiome mock control D6331 (A), Five mouse stool samples (B), Five human stool samples (C), Experiment design (D), PowerLyzer 24 Homogenizer used for homogenization (E). Gut microbiome sequencing using V3-V4 16S rRNA amplicon method on MiSeq, Illumina (F), Full length 16S rRNA gene sequencing on MinlON, Oxford Nanopore (G) and OTU clustering analysis (H). 
ZymoBIOMICS protocol, and 100 ul was eluted for downstream experiments. The DNA concentration was measured using the Picogreen method (Invitrogen Quant-iT ${ }^{\mathrm{TM}}$ Picogreen dsDNA Assay Kit Reference No. P11496 on Perkin Elmer 2030 Multilabel Reader Victor X3), and the DNA integrity number (DIN) was determined on 4150 Tapestation from Agilent using Agilent's gDNA Screen Tape (Reference No. 5067-5365) and Agilent's gDNA Reagents (Reference No. 5067-5366).

\section{$16 S$ rRNA v3-v4 Amplicon Sequencing on MiSeqDx}

We used 10-20 ng of stool-purified DNA to amplify hypervariable region V3-V4 of the bacterial 16S rRNA gene using the Zymo Research Quick-16S NGS Library Prep Kit (Catalog No. D6400). A single amplicon of about 460 bp was amplified using the Quick-16S Primer Set V3-V4 included in the library prep kit. PCR was stopped using the Reaction Clean-Up Solution per the kit's recommendations. Next, Zymo Research Index Primer Set A was ligated to the amplicon for multiplexing. After the addition of barcodes, the final library size was $596 \mathrm{bp}$. Then the libraries were then brought to a volume of $50 \mathrm{ul}$ with the addition of DNase/RNase-free water and purified using $56 \mathrm{ul}$ of Beckman Coulter's AMPure XP beads (Catalog No. A63881). The final libraries were eluted using $27.5 \mathrm{ul}$ of Tris- $\mathrm{HCl}$ buffer (10mM, pH 8.5). Quality and quantity of each sequencing library were assessed using Agilent's 4150 Tapestation using gDNA Screen Tape and gDNA Reagents both from Agilent and picogreen measurements, respectively. The libraries were then pooled in equal concentrations according to picogreen measurements. Each pool was quantified using the KAPA Biosystems Library Quant Kit (illumina) ROX Low qPCR Mix (Reference No. 07960336001) on an Applied Biosystems 7500 Fast Real-Time PCR system. According to the qPCR measurements, $6 \mathrm{pM}$ of pooled libraries was loaded onto a MiSeqDX flow cell and sequenced using MiSeq Reagent Kit v3 600 Cycles PE (Paired end 300 bp). Raw FASTQ files were demultiplexed based on unique barcodes and assessed for quality.

\section{Full Length 16S rRNA Gene Sequencing on MinION}

To determine the impact of bead-beating intensity on specieslevel diversity in the gut microbiome, the full length 16S rRNA gene was sequenced on subset samples (one Zymo, two Mouse, and three human samples) using long-read sequencing technology from Oxford Nanopore. About $20 \mathrm{ng}$ of DNA was used to amplify and barcode the entire $(1.5 \mathrm{~Kb})$ segment of the $16 \mathrm{~S}$ rRNA gene using Nanopore's 16S Barcoding Kit (SQK16S024). The PCR product was then purified using Beckman Coulter's AMPure XP beads (Catalog No. A63881). The purified amplicon libraries were quantified using the Qubit dsDNA HS Assay kit (Catalog No. Q32854) on an Invitrogen Qubit 4 fluorometer. Then sequencing adapters were ligated to the pooled barcoded reads according to the manufacturer's instructions. Sequencing was performed using R9.4.1 flow cell for $24 \mathrm{~h}$ on the MinION (Oxford Nanopore Technologies).
Nanopore sequence data was analyzed with EPI2ME Agent v2020.2.10. The $16 \mathrm{~S}$ sequences were assigned taxonomy using the What's in my pot? (WIMP) workflow as illustrated in Figure 1.

\section{Amplicon and Full Length Sequence Data Analysis}

Samples with more than $50 \mathrm{~K}$ QC pass short sequencing reads from MiSeqDx were used for $16 \mathrm{~S}$ OTU analysis. Taxonomic classification and operational taxonomic units (OTUs) abundance analysis was performed using the CLC Bio microbial genomics module (https://www.qiagenbioinformatics. com/plugins/clc-microbial-genomics-module/). Individual sample reads were annotated with the Greengenes v13 database using a $97 \%$ similarity index. Alpha and beta diversity analysis was done to understand within- and between-treatment group diversity, respectively. Nanopore 16S data were analyzed using the EPI2ME pipeline and WIMP workflow from Oxford Nanopore Technology (ONT). Raw FASTQ files from Illumina and Nanopore sequencing have been deposited in the Sequence Read Archive (SRA) with accession no. PRJNA685188 (v3-v4 amplicon data).

\section{Data Analysis}

To compare the microbiome diversity between samples and treatments, we applied PERMANOVA analysis (PERmutational Multivariate ANalysis Of VAriance, also known as nonparametric MANOVA (Tang et al., 2016) available in CLC Bio Microbial Genomics Module 20.0). This measures effect size and significance on beta diversity for variables. The significance is obtained by a permutation test. In addition, abundances across various bead-beating conditions were compared using a linear model differential abundance test. This tool models each feature (e.g., OUT or an organism) as a separate generalized linear model (GLM). Microbiome compositions were compared across time points using DEseq (DESeq2_1.26.0), in R version 3.6.1. Data in Figures 3-5 represent relative abundance of species determined based on the number of reads detected for that species. Total observed reads for a species were normalized to 1 and then relative abundance at each treatment point was calculated. Statistical analysis was performed using GraphPad Prism software version 8 (GraphPad). T-test was performed and a two-tailed $\mathrm{p}$ value of $<0.05$ was considered significant. MinION sequencing data were analyzed using EPI2ME Agent v2020.2.10; $16 \mathrm{~S}$ sequencing reads were assigned taxonomy using the WIMP workflow.

\section{RESULTS}

\section{Impact of Bead-Beating Intensity on DNA Integrity and Quantity}

As shown in Supplementary Figure 1, total DNA yield was significantly (t-test $\mathrm{p}<0.05)$ high in the samples beaten for 4 and 9 min (Supplementary Figure 1A). DNA integrity number (DIN) was high in samples beaten for 1 or $4 \mathrm{~min}$ 
(Supplementary Figure 1B). The number of pass filter sequencing reads were similar across the treatments (Supplementary Figure 1C). We also compared the total number of high-confidence OTUs annotated in all the samples in the amplicon and full length sequencing data. Median values of these data are compared using a t-test and presented in scatter plots in Supplementary Figure 1D. Overall, the data suggest that bead-beating has no significant impact on the number of sequence reads or OTUs (Supplementary Figure 1D).

\section{Impact of Bead-Beating Intensity on Microbial Diversity}

Quality pass sequencing reads were used to cluster OTUs in the study samples. Supplementary Figure 2 shows v3-v4 amplicon data-based phylum level OTUs in mouse and human stool samples. Alpha and beta diversity indices were determined for various bead-beating intensities (Supplementary Figures 3A, B). As shown in Supplementary Figure 3B, mouse samples were tightly clustered based on bead-beating condition. The Bray-Curtis method of beta-diversity assessment was used to evaluate dissimilarity index between communities. Shannon's entropy and Simpson's indices were reduced upon extensive beadbeating (Supplementary Figures 3C, D). However, overall phylogenetic diversity was higher in 4-min bead-beaten mouse stool (Supplementary Figure 3E). Abundance of OTUs in different bead-beating treatments was compared using generalized linear model differential abundance test on groups defined by bead-beating treatments (Supplementary Table 1). In addition, PERMANOVA analysis was also used to measure significance on beta diversity (Supplementary Table 2). Similar analysis was performed in human stool data and OTUs with statistically different abundances (Supplementary Table 3). Supplementary Figures 3F, G show alpha- and beta diversity analysis in human stool samples. As shown, beta-diversity indices in human stool samples were very different from that of mice, as higher between sample diversity was observed (Supplementary Figure 3G and Supplementary Table 4). We observed high Simpson's index and Shannon entropy as well as phylogenetic diversity in 4- and 9-min bead-beaten samples in human stool (Supplementary Figures $\mathbf{3 H}-\mathbf{J}$ ), respectively. Overall, analysis showed that studied parameters were more consistent for the 4 min group in both mouse and human samples.

\section{Validation of Sequencing and Analysis Pipeline on Mock Control}

We tested and standardized our $16 \mathrm{~S}$ sequencing and data analysis pipeline on a Zymo mock control sample. As shown in Supplementary Figure 4 and Supplementary Table 5, our assay was able to recover all Gram-negative and Gram-positive strains in a mock sample in very similar proportions as pooled by Zymo Research (Pearson $\mathrm{r}=0.75-0.87, \mathrm{p}<0.001$ ), suggesting that our assay was capturing the read out quite accurately. As expected, bead-beating had a relatively moderate impact on the recovery of Gram-negative bacteria such as Escherichia, Prevotella, and Akkermansia (Supplementary Figure 4A). On the other hand, maximum abundance of Gram-positive bacteria such as Roseburia, Bifidobactarium, and Lactobacillus was only captured either in 4- or 9-min beaten samples (Supplementary Figure 4B). Consistent with literature that suggests that the complex cell wall in Gram-positive bacteria requires more intense lysis, we observed a strong correlation (Pearson's $r=0.91$ ) between bead-beating intensity and recovery of Grampositive strains in mock control (Supplementary Figure 4C). We used the same sequencing protocol and analysis pipeline to analyze the gut microbiome in mouse and human stool next.

\section{Bead-Beating Treatment Stratifies Gut Microbes Into Four Major Clusters}

Analysis of $16 \mathrm{~S}$ rRNA v3-v4 amplicon sequencing data in mouse stool showed that the recovery of phylum Actinobacteria and Firmicutes was significantly $(\mathrm{p}<0.05)$ affected by bead-beating intensity (Figure 2A). As illustrated, maximum recovery of Actinobacteria and Firmicutes was observed in samples beaten for 4 and $9 \mathrm{~min}$. On the other hand, the highest abundance of Proteobactaria and Bacteroidetes was observed in unbeaten or 1-min beaten samples (Figure 2A). Consistent with mouse stool data, Actinobacteria, Bacteroidetes, and Proteobacteria in human stool also showed similar sensitivity to bead-beating treatment, however human samples were more heterogeneous (Figure 2B). Interestingly, Firmicutes detected in mouse and human stool exhibited different sensitivity to bead-beating treatment (Figures 2C, D). As shown in Figure 2C, Firmicutes in mice stool were mostly comprised of Allobaculum and Clostridiales and showed a consistent pattern of recovery across all the samples (Supplementary Table 6A). On the other hand, Firmicutes in human stool were comprised of more diverse bacteria that showed large sample-to-sample heterogeneity in composition and abundances (Figure 2D). As shown, Allobaculum in mouse stool required extensive bead-beating for maximum recovery, whereas Firmicutes in human stool, i.e., Veillonella, Ruminococcus, and Acidaminococcus showed maximal recovery in unbeaten or 1-min beaten samples (Supplementary Table 6B). These differences in the composition of Firmicutes in mouse and human stool were the reasons for discordant results. Analysis of top bacteria in mouse stool data showed that recovery of Lactobacillus, Allobaculum, Bifidobacterium, Coriobacteriaceae, F16, and Clostridiales was significantly $(\mathrm{p}<0.05)$ affected by bead-beating treatment (Supplementary Table 7). Similarly, comparison of OTUs in human stool samples revealed the differences in abundances between the four bead-beating conditions (Supplementary Table 8). Analysis showed that recovery of Dorea, Blautia, Ruminococcus, Lactobacillus, and Bifidobacterium was significantly $(\mathrm{p}<0.05)$ affected by bead-beating intensity (Supplementary Table 8). These data suggest that applying the same bead-beating treatment to mouse and human stool samples may obscure the actual diversity of the gut microbiome.

Next, we clustered the genus-level OTUs in mouse and human stool based on their sensitivity to bead-beating intensity. We used ClustVis (Metsalu and Vilo, 2015) to stratify bacteria into clusters based on their abundance at various bead-beating conditions (Figures 2E, F). As shown in Figure 2E, this analysis stratified the top 22 OTUs into four major clusters in mouse stool. Cluster 1 was comprised of bacteria Dorea, Bifidobacterium, Lactobacillus, 

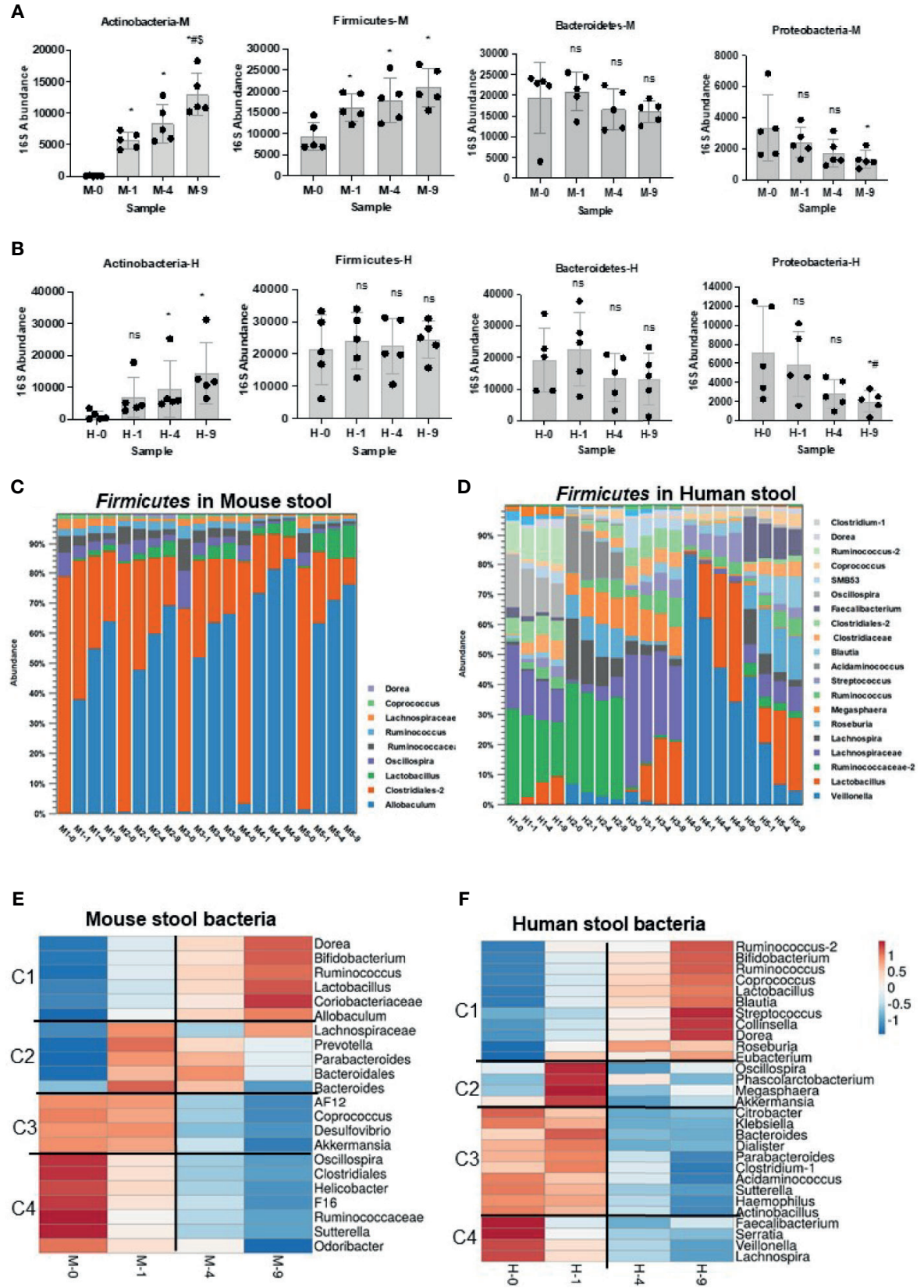

FIGURE 2 | Impact of bead-beating on Phylum- and Genus-level recovery of gut microbiota. Panel (A) Relative abundance of Actinobacteria, Firmicutes, Bacteroidetes, and Proteobacteria at four bead-beating conditions in mouse stool. Panel (B) shows the relative abundance of Actinobacteria, Firmicutes, Bacteroidetes, and Proteobacteria at four bead-beating conditions in human stool. Each black dot represents an individual stool sample. Bar plots show relative $16 S$ abundance on the $y$-axis and samples on the $x$-axis. Panels (C) shows detected abundances of various Firmicutes in mouse stool. Panel (D) shows abundances of Firmicutes in human stool. Panel (E) Heatmap shows four clusters of mouse stool bacteria that differ in their abundances at various bead-beating conditions. Panel (F) Heatmap shows four clusters of human stool bacteria that differ in their abundances at various bead-beating conditions. These clusters were generated using ClustVis, a web tool for visualizing clustering of multivariate data (BETA) (Metsalu and Vilo, 2015). Blue and red color in the heatmap indicate lowest or highest abundance, respectively. Statistical p-values are denoted with ${ }^{*}$ " and ${ }^{\$}$ represent comparison of unbeaten sample data with 1-, 4-, and 9-min beaten samples, respectively. ns, not significant.

and Allobaculum that showed maximum abundance in the 9-min beaten sample as compared to the unbeaten sample. The second cluster included bacteria such as Prevotella and Bacteroides that showed maximum recovery at 1 or 4 min of bead-beating. Cluster 3 included bacteria that required minimal shaking, $1 \mathrm{~min}$ of or no bead-beating at all. The fourth cluster was comprised of organisms such as Helicobacter and Sutterella that showed maximum recovery in the unbeaten sample (Figure 2E). 


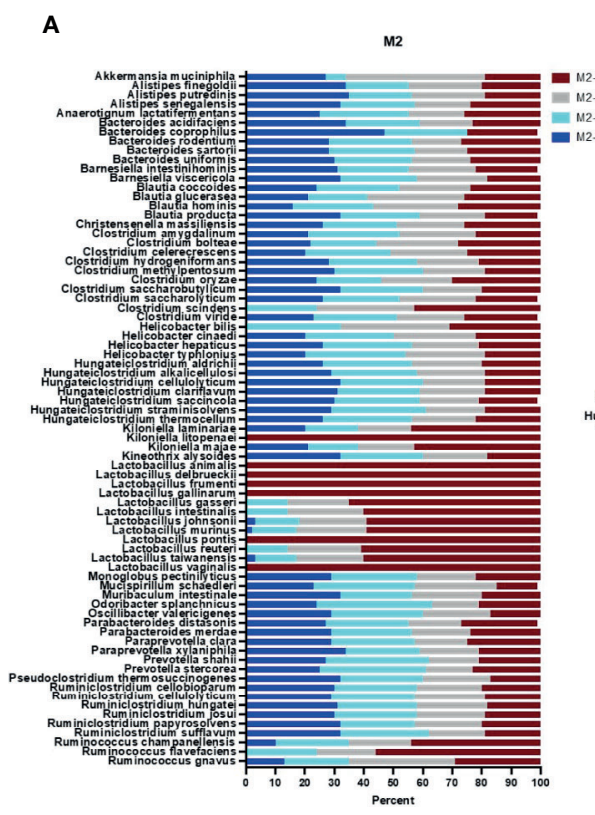

\section{B}

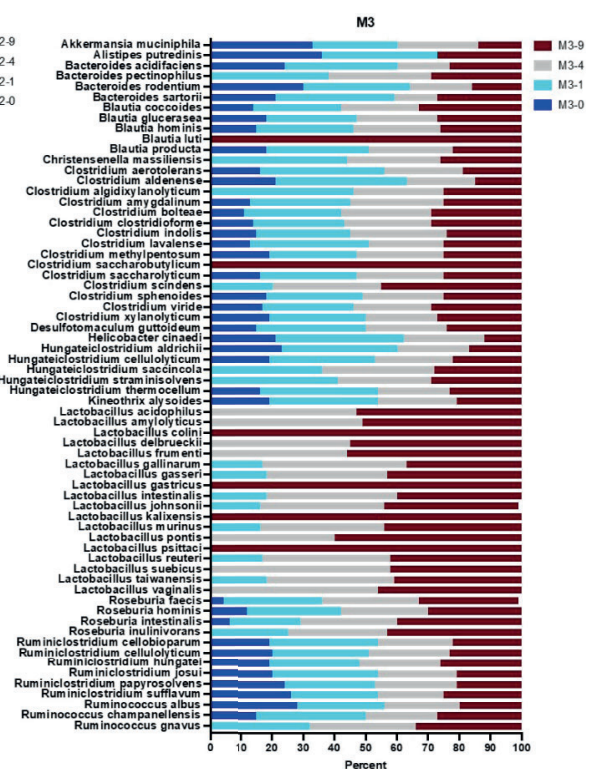

FIGURE 3 | Full length 16S rRNA gene sequence-based taxonomic classification of bacteria in mouse stool samples. Plots in panels (A, B) show relative abundance of detected species at four bead-beating conditions in M2 and M3 stool, respectively. The $x$-axis shows the percentage of reads supporting the taxon and the $y$-axis shows the detected species. In the sample ID, M represent Mouse and $-0,-1,-4$, and -9 represent bead-beating time in the experiment.

A

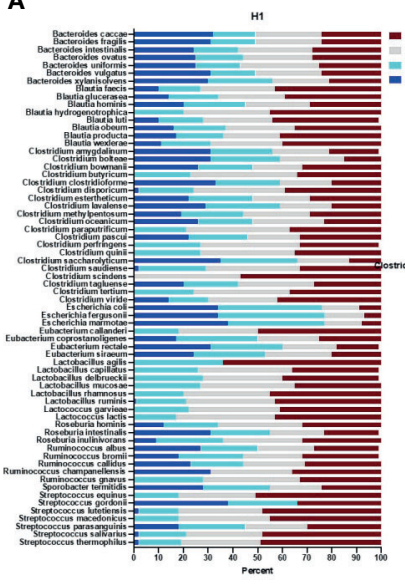

B

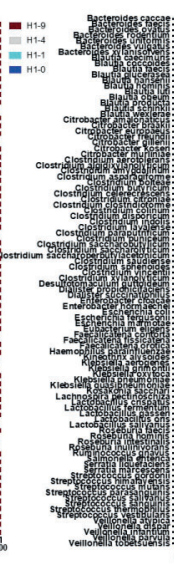

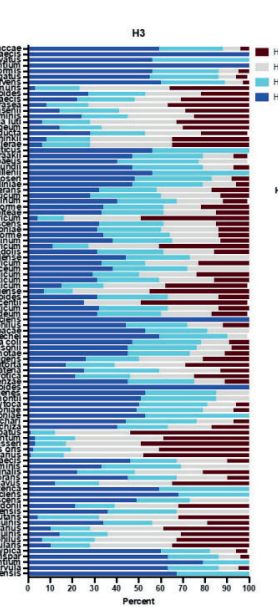

C

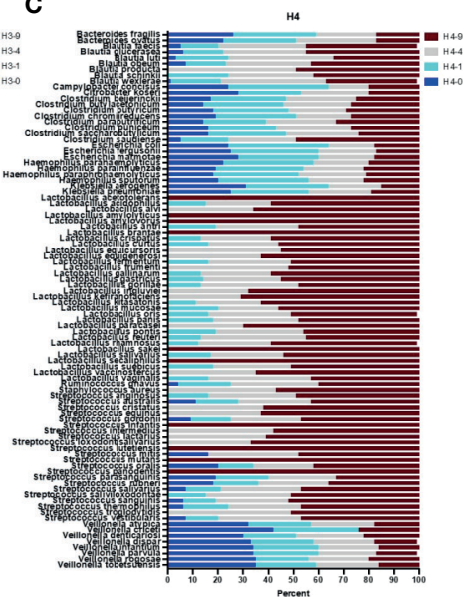

FIGURE 4 | Full length 16S rRNA gene sequence-based taxonomic classification of bacteria in human stool samples. Plots in panels (A-C) show relative abundance of detected species at four bead-beating conditions in $\mathrm{H} 1, \mathrm{H} 3$, and $\mathrm{H} 4$ stool, respectively. The x-axis shows the percentage of reads supporting the taxon and the $\mathrm{y}$-axis shows detected species. In the sample ID, $\mathrm{H}$ represent Human and $-0,-1,-4$, and -9 represent bead-beating time in the experiment.

Similar clusters of bacteria were also observed in human stool as well (Figure 2F). Cluster 1 included many of the common human gut commensals such as Dorea, Blautia, Bifidobacterium, and Lactobacillus. These organisms were very underrepresented in unbeaten samples. Cluster 2 constitutes the moderate group that certainly required some $(1 \mathrm{~min})$ bead-beating treatment as suggested by its reduced recovery in both 0 as well as in 9-min beaten samples. Interestingly, cluster 3 included some known human pathogens such as Klebsiella, Hemophilus, and Citrobacter. These organisms showed maximum recovery in unbeaten or 1-min beaten samples (Figure 2F). Cluster 4 showed maximum recovery of OTUs in unbeaten samples. This cluster included Faecalibacterium, Serratia, Veillonella, and Lachnospira (Figure 2F). 


\section{A}

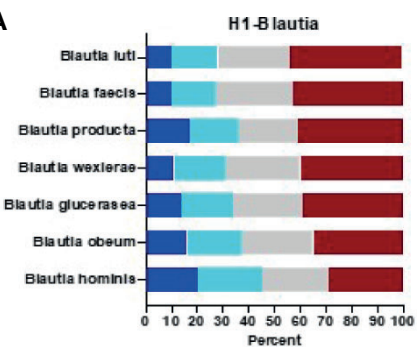

C

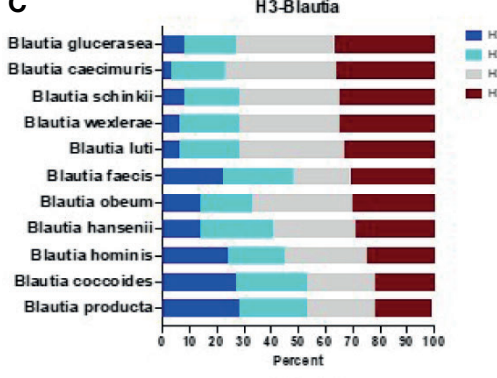

E

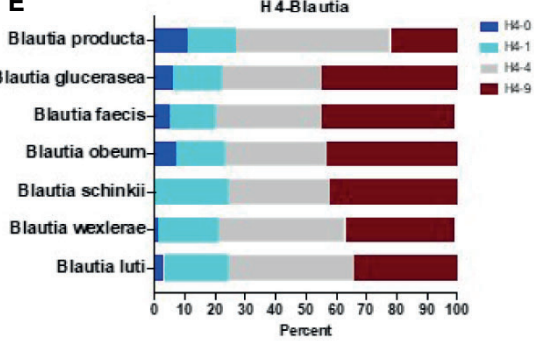

G

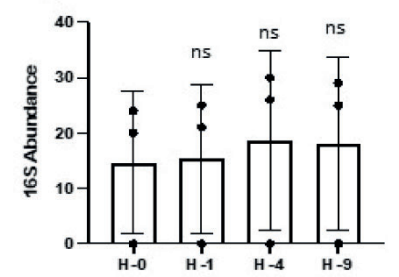

I Streptococcus parasangulnIs

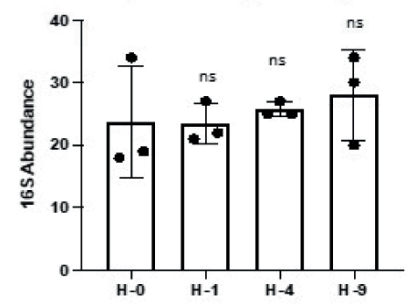

B

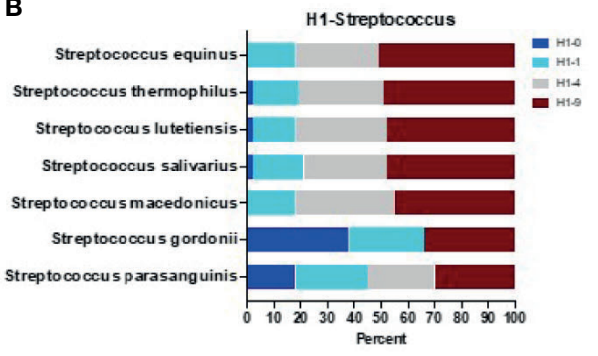

D

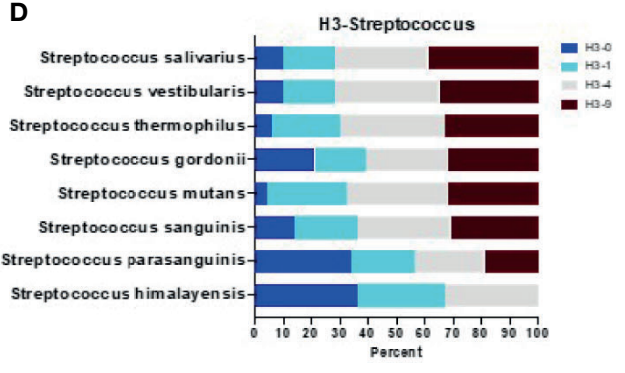

$\mathbf{F}$

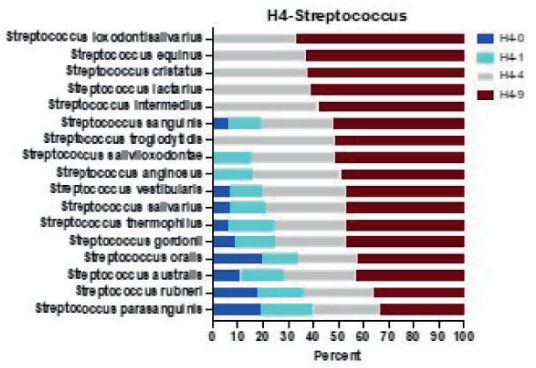

H

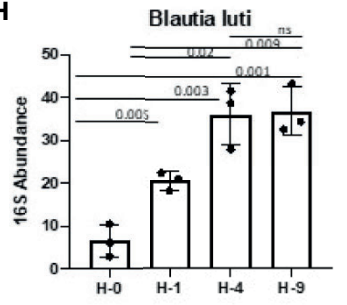

J Streptococcus thermophilus

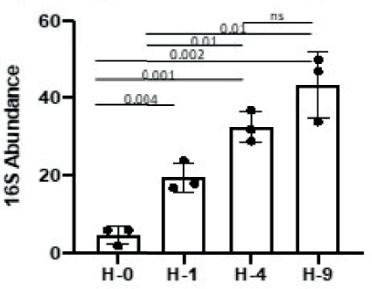

FIGURE 5 | Species of genus Blautia and Streptococcus show differential sensitivity to bead- beating treatment. Panels (A-F) illustrate the abundance of various species of Blautia and Streptococcus at four $(0,1,4$, and $9 \mathrm{~min})$ bead-beating conditions in three human stool samples ( $\mathrm{H} 1$, $\mathrm{H} 3$, $\mathrm{H} 4)$ ). Relative abundance of various species of Blautia and Streptococcus at each condition is plotted in bar graphs. Relative abundance was calculated based on number of full length 165 rRNA sequencing reads supporting a given taxon in each condition. Panel (G) shows abundance of Blautia hominis at four different bead-beating conditions in human stool. Panel (H) shows abundance of Blautia luti at different bead-beating conditions in human stool. Panel (I) shows abundance of Streptococcus parasanguinis at four different bead-beating conditions in human stool. Panel $\mathbf{( J )}$ shows abundance of Streptococcus thermophilus at different bead-beating conditions in human stool. The $y$-axis shows the $16 \mathrm{~S}$ abundance of detected bacteria and the x-axis shows different beating conditions. Numeric data were compared using t-test statistics and a p-value <0.05 was considered significant. "ns" denotes "Not significant". Sample IDs denotes H-0: 0 min, H-1: 1 min, H-4:4 min, and H-9:9 min of bead-beating time. 


\section{Impact of Bead-Beating Intensity on the Species-Level Recovery of the Gut Microbiome}

The $16 \mathrm{~S} \mathrm{v} 3-\mathrm{v} 4$ amplicon sequencing data were very limited in species-level taxonomic classification of detected OTUs (Supplementary Table 9). About $35-40 \%$ of OTUs were classified up to a species level of taxonomy. The remaining 60$65 \%$ were only classified up to the phylum, class, order, family, or genus level (Supplementary Table 10). So, next we performed full length 16S rRNA gene sequencing on two mice (M2, M3) and three human stool samples (H1, H2, H4). Long-read sequencing data was classified into various taxonomic ranks using the EPI2ME WIMP pipeline. NCBI taxonomy trees were generated based on the number of detected reads. The 16s v3-v4 amplicon and full length $16 \mathrm{~S}$ rRNA sequencing data showed good correlation at the phylum level (Pearson $r>0.70, \mathrm{p}<0.01$ ). Full length data characterized vast species-level diversity in mouse and human samples (Figures 3, 4). The $16 \mathrm{~S}$ v3-v4 amplicon data annotated 14 and 10 OTUs to the species level of taxonomy in M2 and M3 mouse stool, respectively. On the other hand, full length sequences-based analysis revealed 98 and 96 bacterial species in M2 and M3 mouse stool, respectively. Similarly, amplicon data in H1, H3, and H4 samples annotated 10,11 , and 8 OTUs to species level respectively, whereas, full length sequence analysis assigned species rank to 155,143 , and 120 detected organisms in $\mathrm{H} 1, \mathrm{H} 3$, and $\mathrm{H} 4$ samples, respectively. These numbers were calculated based on a minimum of 15 long reads to support a taxon. Analysis of full length data showed that about 78\% (75 out of 97) of the total observed species in M2 could be detected in the unbeaten sample, whereas only 59\% (56 out of 95) of the total observed species were detected in the unbeaten M3 stool, suggesting potential differences in the proportion of bacteria that are more sensitive to bead-beating treatment. Next, to calculate the relative percentage of observed species at each time point, we normalized the total reads for the given species to 100 percent. Data presented in Figures 3-5 show normalized relative percentages of observed species. This allowed comparison of recovery in terms of observed abundance of the species at each bead-beating treatment. Furthermore, analysis showed that about 27\% (26 out of 95) of bacteria in M3 stool showed maximum abundance in 4- or 9-min beaten samples as compared to $10 \%$ (10 out of 97 ) in M2 (Figure 3B and Supplementary Figure 5). As shown in Supplementary Figure 5, long-read sequencing data profiled species diversity in several major gut commensal genera, i.e., Bacteroides, Clostridium, Lactobacillus, Ruminococcus. We observed that some species in Roseburia, Blautia, and Ruminococcus showed variation in their recovery with respect to bead-beating treatment. Of these, $R$. gnavus and $R$. albus species of the Ruminococcus genus were particularly interesting as the maximum abundance of $R$. gnavus was detected in 4- and 9-min beaten samples, whereas $R$. albus species showed maximum abundance in 0 - or 1-min beaten mouse samples (Supplementary Figure 5H and Supplementary Table 11).

Next, full length 16S rRNA sequences-based microbiome analysis in three human stool samples $(\mathrm{H} 1, \mathrm{H} 3$, and $\mathrm{H} 4)$ revealed vast species diversity (about an 8-10-fold increase compared to amplicon data) and sample-to-sample variation in the microbiome composition as suggested by variable number and types of detected bacteria (Figure 4 and Supplementary Table 12). The number of bacteria detected in different bead-beating treatments varied from sample to sample (Supplementary Table 12). It was observed that species of the genus Blautia, Streptococcus, and Ruminococcus exhibited variation in recovery with respect to bead-beating treatment (Supplementary Figures 6A-E). Full length sequencing data analysis showed significant diversity and heterogeneity in organisms detected in human stool (Figure 4B). For example, in sample $\mathrm{H} 3$, some clinically relevant microbes such as Citrobacter freundii and Klebsiella pneumoniae were also detected (Supplementary Figures 6F-J). We observed the highest abundance of these bacteria in the unbeaten sample (Supplementary Figures 6F-J). Similarly, species of Lactobacillus and Streptococcus in the $\mathrm{H} 4$ sample consistently showed highest abundance in the 9-min beaten sample (Supplementary Figures 6K-N). Although three samples are not enough for statistical comparisons, these data do explore microbial diversity and sample-to-sample heterogeneity in microbiota composition in human fecal material. Many OTUs in $\mathrm{H} 1$ and $\mathrm{H} 4$ showed maximum abundances in 4-9-min beaten samples. On the other hand, a large number of OTUs in $\mathrm{H} 3$ stool showed maximum abundance in the unbeaten sample (Figure 4 and Supplementary Table 12). Next, we sorted bacterial species based on Gram-positive and Gram-negative classification and assessed their recovery across four bead-beating conditions in $\mathrm{H} 3$ stool. As expected, Gramnegative species showed maximum abundance in the unbeaten sample (Supplementary Figure 7). We compared amplicon OTU composition in mouse and human stool across bead-beating time points using DEseq analysis (Supplementary Tables 13, Supplementary Table 14). We also compared abundances of various species detected in full length $16 \mathrm{~S}$ data analysis across four bead-beating treatments and identified those that showed significant variation in recovery between four conditions (Supplementary Table 15). As shown in Figures 5A-F, we observed variation in recovery of Blautia and Streptococcus species. For example, the abundance of Blautia hominis species showed no significant difference in recovery between the four beadbeating conditions, whereas Blautia luti and other species of this genus showed maximum abundance in the 9-min beaten sample (Figures 5G, H and Supplementary Table 15). Similarly, abundance of Streptococcus parasanguinis species was not significantly different at the four different bead-beating conditions as was the case with Streptococcus thermophilus and other common species of this genus that exhibited significantly $(\mathrm{p}<0.01)$ higher abundance in the 9 -min beaten sample (Figures 5I, J, Supplementary Table 15). These data support our hypothesis that different species of a genus may exhibit variation in sensitivity to bead-beating treatment.

\section{Impact of Bead-Beating on the Recovery of Ruminococcus gnavus vs. Ruminococcus albus}

In mouse stool data, we observed that about $50 \%$ of Ruminococcus reads were annotated as $R$. gnavus that showed 
maximum abundance in 9-min beaten samples in all the mice samples. The remaining reads that could not be assigned any species showed relatively higher abundance in unbeaten or 1min beaten samples, as shown in M2 and M3 (Figure 6A). Full length 16S rRNA sequencing in M2 and M3 samples revealed other species such as Ruminococcus champanellensis, Ruminococcus flavefaciens, and Ruminococcus albus that were rather more abundant than Ruminococcus gnavus (Figure 6B). Interestingly, we observed that abundance of Ruminococcus albus species in M3 long-read data was highest in the unbeaten sample. Similarly, amplicon data on human stool also showed a lot of Ruminococcus reads that were not classified into any species, consistent with mouse stool data. Although maximum abundance of Ruminococcus was captured in 9-min beaten samples, abundance of unclassified species was also observed at 1 and $4 \mathrm{~min}$ as well. The most interesting sample was $\mathrm{H} 1$, in which about $80 \%$ of Rumnicoccus reads could not be classified into any species, whereas the other two samples (H3 \& $\mathrm{H} 4)$ showed a high abundance of $R$. gnavus reads (Figure 6C). Full length $16 \mathrm{~S}$ rRNA gene sequencing in these three samples further revealed the species-level diversity, especially in the $\mathrm{H} 1$ sample. As shown in Figure 6D, the H1 sample showed the presence of various Ruminococcus species including Ruminococcus albus, Ruminococcus bromii, Ruminococcus callidus, Ruminococcus champanellensis as well as Ruminococcus gnavus. More interestingly, consistent with M3 mouse data, the abundance of Ruminococcus albus in the $\mathrm{H} 1$ sample was also highest in the unbeaten sample (Figure 6D). Unlike H1, samples $\mathrm{H} 3$ and $\mathrm{H} 4$ were mostly enriched with Ruminococcus gnavus species, which is consistent with their amplicon data in Figure 6C. Next we performed multisequence alignments and phylogenetic analysis of various Ruminococcus species to explore the $16 \mathrm{~S}$ gene regions of divergences between these species (Figures 6E, F). As shown, complete $16 \mathrm{~S}$ gene sequence alignment between $R$. albus and $R$. gnavus species showed that most of the differences lay outside the v3-v4 hypervariable region (Figure 6E). Maximum likelihood phylogenetic analysis on complete $16 \mathrm{~S}$ rRNA gene sequences showed a genetic relationship between four common Ruminococcus species (Figure 6F).

\section{Impact of Bead-Beating Intensity on the Recovery of Known Human Pathogens}

To assess the impact of bead-beating on the recovery of some clinically relevant microbes, we performed a bead-beating experiment on the ZymoBIOMICS microbial community standard (D6300) that represents a balanced mixture of common infectious microorganisms including Listeria monocytogenes, Bacillus subtilis, Staphylococcus aureus,

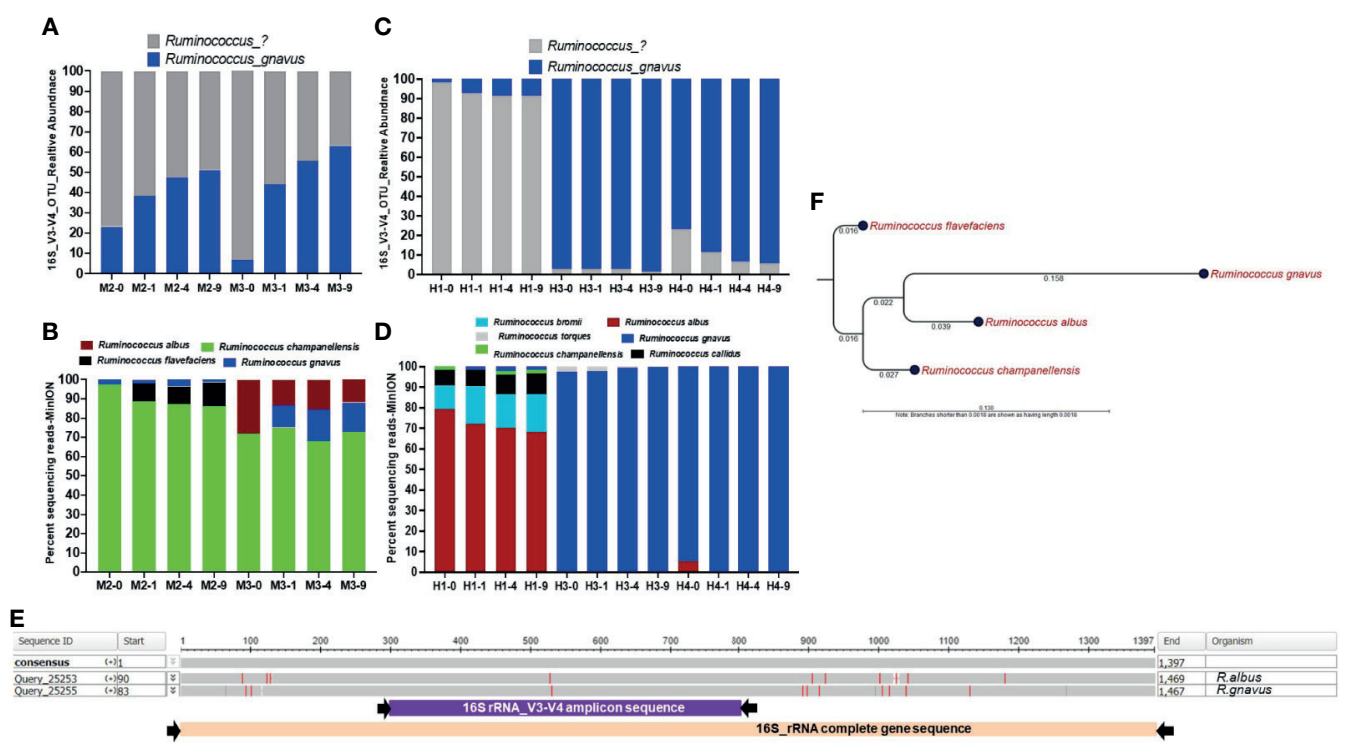

FIGURE 6 | Bead-beating intensity impacts recovery of Ruminococcus species. Panel (A) Bar plots show abundance of Ruminococcus in v3-v4 amplicon data in mouse stool. Panel (B) shows full length 16S rRNA sequencing data in mouse stool. Panel (C) Bar plots show v3-v4 amplicon data-based abundance of Ruminococcus in human stool. Panel (D) shows the proportion of various Ruminococcus species detected by full length $16 \mathrm{~S}$ rRNA sequencing data in human stool. The $\mathrm{x}$-axis shows the sample number and four different bead-beating conditions $0 \mathrm{~min}, 1 \mathrm{~min}, 4 \mathrm{~min}$, and 9 min. The $y$-axis for panels (B, D) shows the percentage of full length $16 \mathrm{~S}$ rRNA sequencing reads that support the detected taxon in the 16S analysis EPI2ME-WIMP pipeline. Panel (E) Nucleotide sequence alignment on the 16S rRNA gene sequence of Ruminococcus albus and Ruminococcus gnavus using NCBl's BLASTn program. The presented image was generated from Multiple Sequence Alignment Viewer 1.20.0. The grey horizontal bar represents conserved 16S rRNA gene sequences and red lines indicate mismatches or differences between two sequences. Purple and pink bars underneath show v3-v4 amplicon and full length $16 S$ rRNA primer-captured gene regions, respectively. Panel (F) 16S rRNA gene sequence-based maximum likelihood phylogenetic tree. Complete 16S rRNA gene sequence of Ruminococcus albus, Ruminococcus gnavus, Ruminococcus champanellensis, and Ruminococcus flavefaciens were aligned and the maximum likelihood phylogenetic tree was constructed using CLC Genomic workbench 12.0.2. Numbers on the tree indicate branch length calculated based on the number of substitutions. 
Enterococcus faecalis, Lactobacillus fermentum, Salmonella enterica, Escherichia coli, and Pseudomonas aeruginosa. The v3v4 amplicon sequencing analysis on this sample showed that Bacillus, Listeria, and Lactobacillus bacteria certainly required 4-9 min of bead-beating for maximal recovery (Supplementary Figures 8A-C). On the other hand, Salmonella, Pseudomonas, and Enterococcus showed maximum recovery in the unbeaten samples (Supplementary Figures 8D-F). The presented data are from two independent experiments on the same Zymo control DNA. Similarly, we also observed a variation in the recovery of clinically relevant microbes in human stool samples. As shown in Supplementary Figures 8G-J, Streptococcus, Dorea, Blautia, and Coporocuccus exhibited a variation in recovery with respect to bead-beating treatment. It was observed that abundance of these bacteria was significantly $(\mathrm{p}=0.05)$ higher in the 9-min beaten sample as compared to the unbeaten sample (Supplementary Figures 8G-J). On the other hand, Hemophilus and Citrobacter showed an opposite trend with maximum recovery in the unbeaten sample (Supplementary Figures $\mathbf{8 K}-\mathbf{N}$ ). However, these results are exploratory and need validation in larger sample cohorts in the future.

\section{DISCUSSION}

Accurate assessment of microbiome structure and composition is very important to study the role of gut microbiota in health and disease (Duvallet et al., 2017). Multiple factors including methods of sample collection, sample storage, DNA extraction, sequencing library preparation, and bioinformatics analysis have been shown to contribute to final microbiome results (Cardona et al., 2012; Carroll et al., 2012; Gorzelak et al., 2015; Rintala et al., 2017; Penington et al., 2018; Proctor et al., 2019). Published literature documents the standards and guidelines for processing and analyzing fecal samples for reproducible microbiome analysis (Santiago et al., 2014). Our assessment of the impact of bead-beating treatment on v3-v4 amplicon and full length $16 \mathrm{~S}$ rRNA sequencingbased analysis of the microbiome reveals the spectrum of species that require minimum or extensive beating for maximum recovery. Observed higher DNA yield and species diversity in 4- and 9-min beaten samples are consistent with published literature (Lim et al., 2018; Teng et al., 2018). Observed maximal recovery of Actinobacteria and Firmicutes in samples subjected to beadbeating for $9 \mathrm{~min}$ is consistent with published reports that show enhanced nucleic acid recovery from Gram-positive organisms with longer disruption of the bacterial cell wall (Yuan et al., 2012). Interestingly, data from the present study showed that bacteria in the mouse and human gut have quantitative variation in sensitivity to bead-beating treatment, as supported by the presence of four different clusters of bacteria. These results suggest that an optimum beating time is necessary to profile the community diversity in a given sample. As also reported by other investigators, our data showed that full length $16 \mathrm{~S}$ rRNA gene sequencing provides high resolution species-level information on gut microbiota (Johnson et al., 2019; Matsuo et al., 2021), which was not achieved with v3-v4 amplicon sequencing. Our analysis suggests that in general various species of a genus show similar sensitivity to bead-beating intensity. However, some species of Ruminococcus, Blautia, Streptococcus, Clostridium, and Roseburia, do seem to exhibit some variability in sensitivity to beating treatment. However, investigations on pure isolates will be needed to validate these observations in future studies. Results in Ruminococcus bacteria are particularly interesting as data suggest that $R$. albus species are more sensitive to bead-beating as compared to R. gnavus. Given that Ruminococcus species are ubiquitous members of the mammalian gastrointestinal tract and play an important role in the digestion of a wide range of plant cell wall polysaccharides, the observed findings are interesting. Furthermore, our study also shows that v3-v4 data were not able to identify Ruminococcus albus species at all. It was full length $16 \mathrm{~S}$ rRNA data that revealed the significant abundance of $R$. albus in the M3 and H1 samples. Precise species-level identification of OTUs is very important as different species may have very different interactions and impact on the host. For example, Ruminococcus albus is a major cellulose degrader in the human gut (Christopherson et al., 2014) and plays an important role in metabolism, on the other hand, blooms of Ruminococcus gnavus has been implicated in autoimmune and inflammatory conditions (Henke et al., 2019). Multiple sequence alignment of all four species of Ruminococcus show that R. albus and R. gnavus, though part of the same clade, are genetically distinct (Figure 6F). More interestingly, complete $16 \mathrm{~S}$ gene sequence alignment between these two species showed that most of the differences lay outside the $\mathrm{v} 3-\mathrm{v} 4$ hypervariable region, which may be the reason that $\mathrm{v} 3-\mathrm{v} 4$ amplicon data could not detect $R$. albus species or distinguish between the two species (Figure 6E). Despite the small sample size, the present study demonstrates the advantage of full length $16 \mathrm{~S}$ rRNA gene sequencing for gut microbiome characterization. Present study data also show that full length 16S rRNA gene sequencing can precisely characterize common and rare species in mouse and human gut microbial communities. Published studies suggest that hundreds of species can co-exist in an individual (Almeida et al., 2019; Yang et al., 2020). So, application of an appropriate DNA extraction method, especially bead-beating intensity, is critical for accurate and comprehensive assessment of species diversity in a sample. In summary, our study demonstrates that the duration of bead-beating has a strong impact on the recovery of common gut commensals as well as clinically relevant microbiota. Our data suggest that a minimum of 4 min of beadbeating (using Qiagen PowerLyzer) can result in recovery of about $70 \%$ of gut microbiota. This study stratifies bacterial species in mouse and human stool that require minimum (0-1 $\mathrm{min})$ or extensive (4-9 $\mathrm{min}$ ) bead-beating for their maximal recovery.

\section{DATA AVAILABILITY STATEMENT}

The datasets presented in this study can be found in online repositories. The names of the repository/repositories and accession number(s) can be found in the article/Supplementary Material. 16S sequencing data (raw FASTQ files) and associated metadata have been deposited in NCBI SRA database with accession no. PRJNA685188. 


\section{ETHICS STATEMENT}

Human stools were collected from healthy volunteers under UT Southwestern Institutional Review Board (IRB) Number STU022011-211. All research protocols and experiment methods used in this study were approved by the IRB. All participants gave their written informed consent to participate in the research.

\section{AUTHOR CONTRIBUTIONS}

$\mathrm{BZ}$ and $\mathrm{MB}$ performed the experiments, CA performed quality control sequencing, $\mathrm{CD}$ provided the mouse stool for the study, $\mathrm{NO}$ and LH contributed to manuscript editing, and PR conceived and designed the experiments and wrote the manuscript. All authors contributed to the article and approved the submitted version.

\section{REFERENCES}

Almeida, A., Mitchell, A. L., Boland, M., Forster, S. C., Gloor, G. B., Tarkowska, A., et al. (2019). A New Genomic Blueprint of the Human Gut Microbiota. Nature 568 (7753), 499-504. doi: 10.1038/s41586-019-0965-1

Bag, S., Saha, B., Mehta, O., Anbumani, D., Kumar, N., Dayal, M., et al. (2016). An Improved Method for High Quality Metagenomics DNA Extraction From Human and Environmental Samples. Sci. Rep. 6, 26775. doi: 10.1038/srep26775

Baker, G. C., Smith, J. J., and Cowan, D. A. (2003). Review and Re-Analysis of Domain-Specific 16s Primers. J. Microbiol Methods 55 (3), 541-555. doi: 10.1016/j.mimet.2003.08.009

Brooks, J. P., Edwards, D. J., Harwich, M. D. Jr., Rivera, M. C., Fettweis, J. M., Serrano, M. G., et al. (2015). The Truth About Metagenomics: Quantifying and Counteracting Bias in 16S rRNA Studies. BMC Microbiol. 15, 66. doi: 10.1186/ s12866-015-0351-6

Cardona, S., Eck, A., Cassellas, M., Gallart, M., Alastrue, C., Dore, J., et al. (2012). Storage Conditions of Intestinal Microbiota Matter in Metagenomic Analysis. BMC Microbiol. 12, 158. doi: 10.1186/1471-2180-12-158

Carroll, I. M., Ringel-Kulka, T., Siddle, J. P., Klaenhammer, T. R., and Ringel, Y. (2012). Characterization of the Fecal Microbiota Using High-Throughput Sequencing Reveals a Stable Microbial Community During Storage. PloS One 7 (10), e46953. doi: 10.1371/journal.pone.0046953

Christopherson, M. R., Dawson, J. A., Stevenson, D. M., Cunningham, A. C., Bramhacharya, S., Weimer, P. J., et al. (2014). Unique Aspects of Fiber Degradation by the Ruminal Ethanologen Ruminococcus Albus 7 Revealed by Physiological and Transcriptomic Analysis. BMC Genomics 15 (1), 1066. doi: 10.1186/1471-2164-15-1066

Costea, P. I., Zeller, G., Sunagawa, S., Pelletier, E., Alberti, A., Levenez, F., et al. (2017). Towards Standards for Human Fecal Sample Processing in Metagenomic Studies. Nat. Biotechnol. 35 (11), 1069-1076. doi: 10.1038/nbt.3960

de Boer, R., Peters, R., Gierveld, S., Schuurman, T., Kooistra-Smid, M., and Savelkoul, P. (2010). Improved Detection of Microbial DNA After BeadBeating Before DNA Isolation. J. Microbiol. Methods 80 (2), 209-211. doi: 10.1016/j.mimet.2009.11.009

Dilhari, A., Sampath, A., Gunasekara, C., Fernando, N., Weerasekara, D., Sissons, C., et al. (2017). Evaluation of the Impact of Six Different DNA Extraction Methods for the Representation of the Microbial Community Associated With Human Chronic Wound Infections Using a Gel-Based DNA Profiling Method. AMB Express 7 (1), 179. doi: 10.1186/s13568-017-0477-Z

Duvallet, C., Gibbons, S. M., Gurry, T., Irizarry, R. A., and Alm, E. J. (2017). MetaAnalysis of Gut Microbiome Studies Identifies Disease-Specific and Shared Responses. Nat. Commun. 8 (1), 1784. doi: 10.1038/s41467-017-01973-8

Falony, G., Joossens, M., Vieira-Silva, S., Wang, J., Darzi, Y., Faust, K., et al. (2016). Population-Level Analysis of Gut Microbiome Variation. Science 352 (6285), 560-564. doi: 10.1126/science.aad3503

\section{FUNDING}

This study was supported by the UT Southwestern Microbiome Research Laboratory.

\section{ACKNOWLEDGMENTS}

Authors gratefully acknowledge donors of de-identified human stool samples for the study.

\section{SUPPLEMENTARY MATERIAL}

The Supplementary Material for this article can be found online at: https://www.frontiersin.org/articles/10.3389/fcimb.2021.678522/ full\#supplementary-material

Fiedorova, K., Radvansky, M., Nemcova, E., Grombirikova, H., Bosak, J., Cernochova, M., et al. (2019). The Impact of DNA Extraction Methods on Stool Bacterial and Fungal Microbiota Community Recovery. Front. Microbiol. 10, 821. doi: 10.3389/fmicb.2019.00821

Gill, S. R., Pop, M., Deboy, R. T., Eckburg, P. B., Turnbaugh, P. J., Samuel, B. S., et al. (2006). Metagenomic Analysis of the Human Distal Gut Microbiome. Science 312 (5778), 1355-1359. doi: 10.1126/science.1124234

Gill, C., van de Wijgert, J. H., Blow, F., and Darby, A. C. (2016). Evaluation of Lysis Methods for the Extraction of Bacterial DNA for Analysis of the Vaginal Microbiota. PloS One 11 (9), e0163148. doi: 10.1371/journal. pone.0163148

Gorzelak, M. A., Gill, S. K., Tasnim, N., Ahmadi-Vand, Z., Jay, M., and Gibson, D. L. (2015). Methods for Improving Human Gut Microbiome Data by Reducing Variability Through Sample Processing and Storage of Stool. PloS One 10 (8), e0134802. doi: 10.1371/journal.pone.0134802

Henke, M. T., Kenny, D. J., Cassilly, C. D., Vlamakis, H., Xavier, R. J., and Clardy, J. (2019). Ruminococcus Gnavus, a Member of the Human Gut Microbiome Associated With Crohn's Disease, Produces an Inflammatory Polysaccharide. Proc. Natl. Acad. Sci. U.S.A. 116 (26), 12672-12677. doi: 10.1073/pnas. 1904099116

Huttenhower, C., Dirk, G., Knight, R., Abubucker, S., Badger, J. H., Chinwalla, A. T., et al. (2012). Structure, Function and Diversity of the Healthy Human Microbiome. Nature 486 (7402), 207-214. doi: 10.1038/nature11234

Janda, J. M., and Abbott, S. L. (2007). 16s rRNA Gene Sequencing for Bacterial Identification in the Diagnostic Laboratory: Pluses, Perils, and Pitfalls. J. Clin. Microbiol. 45 (9), 2761-2764. doi: 10.1128/JCM.01228-07

Jenkins, C., Dallman, T. J., and Grant, K. A. (2019). Impact of Whole Genome Sequencing on the Investigation of Food-Borne Outbreaks of Shiga ToxinProducing Escherichia Coli Serogroup O157:H7, England, 2013 to 2017. Euro Surveill. 24 (4):1800346. doi: 10.2807/1560-7917.ES.2019. 24.4.1800346

Johnson, J. S., Spakowicz, D. J., Hong, B. Y., Petersen, L. M., Demkowicz, P., Chen, L., et al. (2019). Evaluation of $16 \mathrm{~S}$ rRNA Gene Sequencing for Species and Strain-Level Microbiome Analysis. Nat. Commun. 10 (1), 5029. doi: 10.1038/ s41467-019-13036-1

Kim, S. J., Chang, J., and Singh, M. (2015). Peptidoglycan Architecture of GramPositive Bacteria by Solid-State NMR. Biochim. Biophys. Acta 1848 (1 Pt B), 350-362. doi: 10.1016/j.bbamem.2014.05.031

Lim, M. Y., Song, E. J., Kim, S. H., Lee, J., and Nam, Y. D. (2018). Comparison of DNA Extraction Methods for Human Gut Microbial Community Profiling. Syst. Appl. Microbiol. 41 (2), 151-157. doi: 10.1016/j.syapm.2017.11.008

Matijašić, M., Meštrović, T., Paljetak, H., Perić, M., Barešić, A., and Verbanac, D. (2020). Gut Microbiota Beyond Bacteria-Mycobiome, Virome, Archaeome, and Eukaryotic Parasites in IBD. Int. J. Mol. Sci. 21 (8), 2668. doi: 10.3390/ ijms 21082668 
Matsuo, Y., Komiya, S., Yasumizu, Y., Yasuoka, Y., Mizushima, K., Takagi, T., et al. (2021). Full-Length 16s rRNA Gene Amplicon Analysis of Human Gut Microbiota Using Minion ${ }^{\mathrm{TM}}$ Nanopore Sequencing Confers Species-Level Resolution. BMC Microbiol. 21 (1), 35. doi: 10.1186/s12866-021-02094-5

Metsalu, T., and Vilo, J. (2015). Clustvis: A Web Tool for Visualizing Clustering of Multivariate Data Using Principal Component Analysis and Heatmap. Nucleic Acids Res. 43 (W1), W566-W570. doi: 10.1093/nar/gkv468

Momozawa, Y., Deffontaine, V., Louis, E., and Medrano, J. F. (2011). Characterization of Bacteria in Biopsies of Colon and Stools by High Throughput Sequencing of the V2 Region of Bacterial 16s rRNA Gene in Human. PloS One 6 (2), e16952. doi: 10.1371/journal.pone.0016952

Nash, A. K., Auchtung, T. A., Wong, M. C., Smith, D. P., Gesell, J. R., Ross, M. C., et al. (2017). The Gut Mycobiome of the Human Microbiome Project Healthy Cohort. Microbiome 5 (1), 153. doi: 10.1186/s40168-017-0373-4

Nelson, K. E., Weinstock, G. M., Highlander, S. K., Worley, K. C., Creasy, H. H., Wortman, J. R., et al. (2010). A Catalog of Reference Genomes From the Human Microbiome. Science 328 (5981), 994-999. doi: 10.1126/ science. 1183605

Penington, J. S., Penno, M. A. S., Ngui, K. M., Ajami, N. J., Roth-Schulze, A. J., Wilcox, S. A., et al. (2018). Influence of Fecal Collection Conditions and 16S rRNA Gene Sequencing at Two Centers on Human Gut Microbiota Analysis. Sci. Rep. 8 (1), 4386. doi: 10.1038/s41598-018-22491-7

Proctor, L., LoTempio, J., Marquitz, A., Daschner, P., Xi, D., Flores, R., et al. (2019). A Review of 10 Years of Human Microbiome Research Activities at the US National Institutes of Health, Fiscal Years 2007-2016. Microbiome 7 (1), 31. doi: 10.1186/s40168-019-0620-y

Qin, J., Li, R., Raes, J., Arumugam, M., Burgdorf, K. S., Manichanh, C., et al. (2010). A Human Gut Microbial Gene Catalogue Established by Metagenomic Sequencing. Nature 464 (7285), 59-65. doi: 10.1038/nature08821

Rintala, A., Pietila, S., Munukka, E., Eerola, E., Pursiheimo, J. P., Laiho, A., et al. (2017). Gut Microbiota Analysis Results are Highly Dependent on the 16S rRNA Gene Target Region, Whereas the Impact of DNA Extraction is Minor. J. Biomol Tech 28 (1), 19-30. doi: 10.7171/jbt.17-2801-003

Santiago, A., Panda, S., Mengels, G., Martinez, X., Azpiroz, F., Dore, J., et al. (2014). Processing Faecal Samples: A Step Forward for Standards in Microbial Community Analysis. BMC Microbiol. 14, 112. doi: 10.1186/ 1471-2180-14-112

Sinha, R., Abu-Ali, G., Vogtmann, E., Fodor, A. A., Ren, B., Amir, A., et al. (2017). Assessment of Variation in Microbial Community Amplicon Sequencing by the Microbiome Quality Control (MBQC) Project Consortium. Nat. Biotechnol. 35 (11), 1077-1086. doi: 10.1038/nbt.3981

Tang, Z. Z., Chen, G., and Alekseyenko, A. V. (2016). PERMANOVA-S: Association Test for Microbial Community Composition That Accommodates Confounders and Multiple Distances. Bioinformatics 32 (17), 2618-2625. doi: 10.1093/ bioinformatics/btw311

Teng, F., Darveekaran Nair, S. S., Zhu, P., Li, S., Huang, S., Li, X., et al. (2018). Impact of DNA Extraction Method and Targeted 16s-rRNA Hypervariable Region on Oral Microbiota Profiling. Sci. Rep. 8 (1), 16321. doi: 10.1038/ s41598-018-34294-x
The Integrative HMP (iHMP) Research Network Consortium. (2019). The Integrative Human Microbiome Project. Nature 569 (7758), 641-648. doi: 10.1038/s41586-019-1238-8

Tremblay, J., Singh, K., Fern, A., Kirton, E. S., He, S., Woyke, T., et al. (2015). Primer and Platform Effects on 16S rRNA Tag Sequencing. Front. Microbiol. 6, 771. doi: $10.3389 /$ fmicb.2015.00771

Valentini, A., Taberlet, P., Miaud, C., Civade, R., Herder, J., Thomsen, P. F., et al. (2016). Next-Generation Monitoring of Aquatic Biodiversity Using Environmental DNA Metabarcoding. Mol. Ecol. 25 (4), 929-942. doi: 10.1111/mec.13428

von Wintzingerode, F., Gobel, U. B., and Stackebrandt, E. (1997). Determination of Microbial Diversity in Environmental Samples: Pitfalls of PCR-Based rRNA Analysis. FEMS Microbiol. Rev. 21 (3), 213-229. doi: 10.1111/j.15746976.1997.tb00351.x

Werner, J. J., Zhou, D., Caporaso, J. G., Knight, R., and Angenent, L. T. (2012). Comparison of Illumina Paired-End and Single-Direction Sequencing for Microbial 16s rRNA Gene Amplicon Surveys. ISME J. 6 (7), 1273-1276. doi: 10.1038/ismej.2011.186

Willner, D., Daly, J., Whiley, D., Grimwood, K., Wainwright, C. E., and Hugenholtz, P. (2012). Comparison of DNA Extraction Methods for Microbial Community Profiling With an Application to Pediatric Bronchoalveolar Lavage Samples. PloS One 7 (4), e34605. doi: 10.1371/journal.pone.0034605

Wu, G. D., Lewis, J. D., Hoffmann, C., Chen, Y. Y., Knight, R., Bittinger, K., et al. (2010). Sampling and Pyrosequencing Methods for Characterizing Bacterial Communities in the Human Gut Using 16s Sequence Tags. BMC Microbiol. 10, 206. doi: 10.1186/1471-2180-10-206

Yang, J., Pu, J., Lu, S., Bai, X., Wu, Y., Jin, D., et al. (2020). Species-Level Analysis of Human Gut Microbiota With Metataxonomics. Front. Microbiol. 11, 2029. doi: 10.3389/fmicb.2020.02029

Yuan, S., Cohen, D. B., Ravel, J., Abdo, Z., and Forney, L. J. (2012). Evaluation of Methods for the Extraction and Purification of DNA From the Human Microbiome. PloS One 7 (3), e33865. doi: 10.1371/journal.pone.0033865

Conflict of Interest: The authors declare that the research was conducted in the absence of any commercial or financial relationships that could be construed as a potential conflict of interest.

Publisher's Note: All claims expressed in this article are solely those of the authors and do not necessarily represent those of their affiliated organizations, or those of the publisher, the editors and the reviewers. Any product that may be evaluated in this article, or claim that may be made by its manufacturer, is not guaranteed or endorsed by the publisher.

Copyright (c) 2021 Zhang, Brock, Arana, Dende, van Oers, Hooper and Raj. This is an open-access article distributed under the terms of the Creative Commons Attribution License (CC BY). The use, distribution or reproduction in other forums is permitted, provided the original author(s) and the copyright owner(s) are credited and that the original publication in this journal is cited, in accordance with accepted academic practice. No use, distribution or reproduction is permitted which does not comply with these terms. 